\title{
HSP90 inhibitors diminish PDGF-BB-induced migration of osteoblasts via suppression of p44/p42 MAP kinase
}

Tetsu Kawabata ${ }^{1,2,3}$, Haruhiko Tokuda ${ }^{2,4}$, Kazuhiko Fujita ${ }^{1,2}$, Rie Matsushima-Nishiwaki ${ }^{2}$, Go Sakai ${ }^{1,2}$, Junko TACHI ${ }^{2,6}$, Tomoyuki Hioki ${ }^{2,5}$, Woo Kim ${ }^{2,6}$, Hiroki IidA ${ }^{6}$, Takanobu OtsukA ${ }^{1}$, and Osamu KozawA ${ }^{2}$

${ }^{1}$ Department of Orthopedic Surgery, Nagoya City University Graduate School of Medical Sciences, Nagoya, Japan; ${ }^{2}$ Department of Pharmacology, Gifu University Graduate School of Medicine, Gifu, Japan; ${ }^{3}$ Department of Orthopedic Surgery, Toyokawa City Hospital, Toyokawa, Japan; ${ }^{4}$ Department of Clinical Laboratory/Medical Genome Center Biobank, National Center for Geriatrics and Gerontology, Obu, Japan; ${ }^{5}$ Department of Dermatology, Kizawa Memorial Hospital, Minokamo, Japan; and ${ }^{6}$ Department of Anesthesiology and Pain Medicine, Gifu University Graduate School of Medicine, Gifu, Japan

(Received 6 June 2019; and accepted 15 June 2019)

\begin{abstract}
Migration of osteoblasts to the sites resorbed by osteoclasts is an essential step in bone remodeling. However, the exact mechanism of osteoblast migration is still not known. We have shown that platelet-derived growth factor (PDGF)-BB induces the migration of osteoblast-like MC3T3-E1 cells through the activation of p38 mitogen-activated protein (MAP) kinase, c-Jun N-terminal kinase (JNK) and p44/p42 MAP kinase. Evidence is accumulating that heat shock protein 90 (HSP90) acts as a central regulator of proteostasis under stress conditions and physiological cell functions. In the present study, using transwell cell migration assay and wound-healing assay, we investigated the involvement of HSP90 in the PDGF-BB-stimulated migration of MC3T3-E1 cells, and the underlying signaling mechanism estimated by Western blot analyses. Onalespib, an HSP90 inhibitor, significantly reduced the PDGF-BB-stimulated migration evaluated by the two types of migration assays. The cell migration was also suppressed by geldanamycin, another type of HSP90 inhibitor. Onalespib markedly attenuated the PDGF-BB-elicited phosphorylation of p44/ p42 MAP kinase without affecting that of p38 MAP kinase or JNK. In addition, the phosphorylation of p44/p42 MAP kinase by PDGF-BB was reduced by geldanamycin. Taken together, these results strongly suggest that HSP90 inhibitors suppress the PDGF-BB-induced osteoblast migration through the attenuation of p44/p42 MAP kinase activity.
\end{abstract}

Bone tissue is continuously regenerated, and the mass and the quality of bone is properly maintained (3). The bone remodeling is strictly regulated by a sophisticated balance between osteoblastic bone formation and osteoclastic bone resorption $(15,22)$. The bone remodeling is initiated with bone resorption by osteoclasts $(11,22)$. Subsequently, osteoblasts migrate to the sites resorbed by osteoclasts,

Address correspondence to: Dr. Osamu Kozawa, Department of Pharmacology, Gifu University Graduate School of Medicine, Gifu 501-1194, Japan

Tel: +81-58-230-6214, Fax: +81-58-230-6215

E-mail: okkasugai@yahoo.co.jp and then start bone formation $(11,22)$. The impairment of balance between bone resorption and bone formation is considered to cause metabolic bone diseases including osteoporosis. Evidence is accumulating that osteoblast migration is an essential step for the bone remodeling and bone fracture repair (21, 23). It has been shown that platelet-derived growth factor (PDGF) is one of the facilitating factors in osteoblast migration (7). PDGF, which is a potent mitogenic growth factor for connective tissue cells including osteoblasts, is recognized to be embedded in the bone matrix $(1,2,6)$. With regard to the PDGF-effect on osteoblast migration, we have shown that PDGF-BB induces the migration of osteoblast- 
like MC3T3-E1 cells through the activation of p38 mitogen-activated (MAP) kinase, c-Jun N-terminal kinase (JNK) and p44/p42 MAP kinase (8). However, the details underlying PDGF-effect on osteoblast migration have not yet been clarified.

Heat shock proteins (HSPs) consist of a superfamily as proteins induced by stressful extracellular circumstances such as heat and hypoxia (9). It is generally recognized that HSPs as molecular chaperones, regulate proteostasis under stress conditions $(9,20)$. Although HSPs have been named according to its molecular mass, the family of HSPs is currently classified into several groups including HSPA (HSP70), HSPB (small HSPs) and HSPC (HSP90) based on the systematic gene symbols (9). Accumulating evidence indicates that HSP90 among the member of HSPs family plays a pivotal role not only in the stress conditions but also in physiological cellular conditions, since HSP90 is ubiquitously expressed at high levels in a variety of quiescent cells including osteoblasts (20). Under the physiological conditions, HSP90 regulates the function of steroid hormone receptors for glucocorticoid and estrogen (20). As for the role of HSP90 in bone metabolism, an HSP90 inhibitor, 17-AAG, reportedly strengthens osteoclastogenesis and facilitates bone metastasis of breast cancer (19). Additionally, it has been shown that geldanamycin, another inhibitor of HSP90, elicits apoptosis and autophagy of osteosarcoma cells (17). We have recently demonstrated that HSP90 inhibitors amplify prostaglandin F $2 \alpha$-induced synthesis of interleukin- 6 , a potent bone remodeling cytokine in osteoblast-like MC3T3-E1 cells (4). However, the exact roles of HSP90 in the functions of osteoblasts are still not known.

In the present study, we investigated whether HSP90 is implicated in the PDGF-BB-induced migration of osteoblast-like MC3T3-E1 cells. We herein showed that HSP90 inhibitors reduced the PDGF-BB-stimulated migration of MC3T3-E1 cells via the downregulation of $\mathrm{p} 44 / \mathrm{p} 42$ MAP kinase.

\section{MATERIALS AND METHODS}

Materials. PDGF-BB was obtained from R\&D system, Inc. (Minneapolis, MN, USA). Onalespib was obtained from Selleckchem (Houston, TX, USA). Geldanamycin was purchased from Sigma-Aldrich Co. (St. Louis, MO, USA). Phospho-specific p38 MAP kinase antibodies, p38 MAP kinase antibodies, phospho-specific JNK antibodies, JNK antibodies, phospho-specific p44/p42 MAP kinase antibodies and $\mathrm{p} 44 / \mathrm{p} 42$ MAP kinase antibodies were purchased from Cell Signaling Technology, Inc. (Beverly, MA, USA). The ECL Western blot detection system was obtained from GE Healthcare Life Sciences (Little Chalfont, UK). Other materials and chemicals were purchased from commercial sources. Onalespib and geldanamycin were dissolved in dimethyl sulfoxide, and the maximum concentration of dimethyl sulfoxide was $0.1 \%$, which did not affect cell migration assay and the detection of the protein level using Western blotting.

Cell culture. Osteoblast-like MC3T3-E1 cells, immortalized clonal cells which have been established from neonatal mouse calvaria (24), were maintained as previously reported (13). Briefly, MC3T3-E1 cells were cultured in $10 \%$ fetal bovine serum (FBS)-containing $\alpha$-minimum essential medium $(\alpha-\mathrm{MEM})$ at $37^{\circ} \mathrm{C}$ in a humidified atmosphere of $5 \% \mathrm{CO}_{2} / 95 \%$ air. The cells were seeded into $90-\mathrm{mm}$ diameter dishes $\left(2 \times 10^{5}\right.$ cells/dish $)$ in $\alpha$-MEM containing $10 \%$ FBS. For cell migration assay, the cultured cells in $\alpha$-MEM containing 10\% FBS for 3 days were sub-cultured in $\alpha$-MEM containing $0.3 \%$ FBS for $6 \mathrm{~h}$, and then applied for the experiments. For Western blotting, the medium was exchanged for $\alpha$-MEM containing $0.3 \%$ FBS after 5 days. The cells were then applied for a Western blot analysis after $48 \mathrm{~h}$.

Cell migration assay. A transwell cell migration assay was performed as previously described in the method of Karagiosis (10) using a Boyden chamber (polycarbonate membrane with 8 - $\mu \mathrm{m}$ pores; Transwell $^{\circledR}$; Corning Costar Corp, Cambridge, MA, USA). In brief, the cultured MC3T3-E1 cells were seeded $\left(10 \times 10^{4}\right.$ cells/well $)$ onto the upper chamber in $\alpha$-MEM containing $0.3 \%$ FBS. The cells were pretreated with onalespib or geldanamycin for $60 \mathrm{~min}$ in the upper chamber, and PDGF-BB was then added to the lower chamber in $\alpha$-MEM containing $0.3 \%$ FBS, and the cells were incubated for $16 \mathrm{~h}$ at $37^{\circ} \mathrm{C}$. The cells on the upper surface of the membrane were then mechanically removed. The migrated cells adherent to the underside of the membrane were fixed with $4 \%$ paraformaldehyde and stained with 4',6-diamidino-2-phenylindole (DAPI) solution for nuclei. The migrated cells were photographed and counted using fluorescent microscopy at a magnification of $20 \times$ by counting the stained cells from three randomly chosen high-power fields.

For a wound-healing assay, the cultured MC3T3-E1 cells were seeded at $10 \times 10^{4}$ cells/well into an Ibidi Culture-Insert 2 Well (Ibidi, Martinsried, Germany) 
with a $500-\mu \mathrm{m}$ margin from the side of the well and allowed to grow for $24 \mathrm{~h}$. After the insert was removed, the cells were pretreated with onalespib or geldanamycin for $60 \mathrm{~min}$, and then stimulated by PDGF-BB for $8 \mathrm{~h}$. The cells were photographed using an EOS Kiss X4 digital camera (Cannon, Tokyo, Japan) connected to CK40 culture microscope (Olympus Optical Co. Ltd., Tokyo, Japan) before the stimulation of PDGF-BB and after $8 \mathrm{~h}$. The area of migrated cells was measured by ImageJ software (version 1.48; NIH, Bethesda, MD, USA).

Western blot analysis. The cultured MC3T3-E1 cells were pretreated with onalespib or geldanamycin for $60 \mathrm{~min}$, and then stimulated by PDGF-BB or vehicle in $1 \mathrm{~mL}$ of $\alpha$-MEM containing $0.3 \%$ FBS for the indicated periods. The cells were then lysed, homogenized and sonicated in a lysis buffer containing $62.5 \mathrm{mM}$ Tris/HCl, pH 6.8, 2\% sodium dodecyl sulfate (SDS), $50 \mathrm{mM}$ dithiothreitol and $10 \%$ glycerol. SDS-polyacrylamide gel electrophoresis (PAGE) was performed by the method of Laemmli (16) in $10 \%$ polyacrylamide gels. The protein was fractionated and transferred onto an Immun-Blot polyvinylidine difluoride (PVDF) membrane (Bio-Rad, Hercules, CA, USA). The PVDF membranes were blocked with $5 \%$ fat-free dry milk in Tris-buffered saline-Tween (TBS-T; $20 \mathrm{mM}$ Tris/HCl, pH 7.6, $137 \mathrm{mM} \mathrm{NaCl}$, $0.1 \%$ Tween 20 ) for $1 \mathrm{~h}$ before incubation with the indicated primary antibodies. A Western blot analysis was performed in a manner according to our previous description (12) using antibodies against phospho-specific p38 MAP kinase, p38 MAP kinase, phospho-specific JNK, JNK, phospho-specific p44/ p42 MAP kinase or p44/p42 MAP kinase as primary antibodies with peroxidase-labeled antibodies raised in goat against rabbit IgG (KPL, Inc., Gaitherburg, MD, USA) being used as secondary antibodies. The primary and secondary antibodies were diluted to optimal concentrations with $5 \%$ fat-free dry milk in TBS-T. The peroxidase activity on the PVDF membrane was visualized on X-ray film using an ECL Western blotting detection system. Each protein was detected on different gels.

Densitometric analysis. A densitometric analysis of the Western blots was carried out using a scanner and image analysis software program (ImageJ version $1.49, \mathrm{NIH})$. The phosphorylated levels were calculated as follows: the background-subtracted signal intensity of each phosphorylation signal was normalized to the respective intensity of total protein, and plotted as the fold increase in comparison to that of the control cells without stimulation.

Statistical analysis. The data were analyzed by an analysis of variance (ANOVA) followed by Bonferroni method for multiple comparisons between pairs, and $P<0.05$ was considered to be statistically significant. The data are presented as the mean \pm SEM of triplicate determinations from three independent cell preparations.

\section{RESULTS}

Effect of onalespib on the PDGF-BB-induced migration of MC3T3-E1 cells

Recent study from our laboratory demonstrated that PDGF-BB induces the migration of osteoblast-like MC3T3-E1 cells evaluated by a transwell cell migration assay (8). To clarify whether HSP90 is implicated in the PDGF-BB-induced migration of MC3T3-E1 cells, we examined the effect of onalespib, an HSP90 inhibitor (3), on the migration using a Boyden chamber. Onalespib significantly suppressed the PDGF-BB (3 ng/mL)-induced migration (Fig. 1). The inhibitory effect of onalespib was dose dependent over the range $0.1-0.5 \mu \mathrm{M}$ (Fig. 1). Onalespib $(0.5 \mu \mathrm{M})$ caused approximately $80 \%$ suppression in the effect of PDGF-BB.

We next examined the effect of onalespib on the MC3T3-E1 cells with a wound-healing assay. We found that the filled area of migrated cells induced by PDGF-BB $(3 \mathrm{ng} / \mathrm{mL})$ reached almost $100 \%$ by $8 \mathrm{~h}$ after the stimulation. The increase of filled area by PDGF-BB $(3 \mathrm{ng} / \mathrm{mL})$ was significantly suppressed by onalespib $(0.3 \mu \mathrm{M})$, which led to about $65 \%$ inhibition in the PDGF-BB-effect (Fig. 2). We previously reported that HSP90 is constitutively expressed in these cells (14), and confirmed that the expression was hardly affected by PDGF-BB (data not shown).

Effect of geldanamycin on the PDGF-BB-induced migration of MC3T3-E1 cells

Additionally, we examined the effect of geldanamycin, another type of HSP90 inhibitor structurally different from onalespib (18), on the PDGF-BBinduced migration of osteoblast-like MC3T3-E1 cells. The PDGF-BB (3 ng/mL)-induced migration of MC3T3-E1 cells assessed by a Boyden chamber was significantly reduced by geldanamycin $(0.3 \mu \mathrm{M})$, which led to a $60 \%$ inhibition in the effect of PDGF-BB (Fig. 3). Furthermore, we examined the effect of geldanamycin on the MC3T3-E1 cell migration by PDGF-BB using a wound-healing assay. The PDGF-BB-stimulated increase of filled area was 

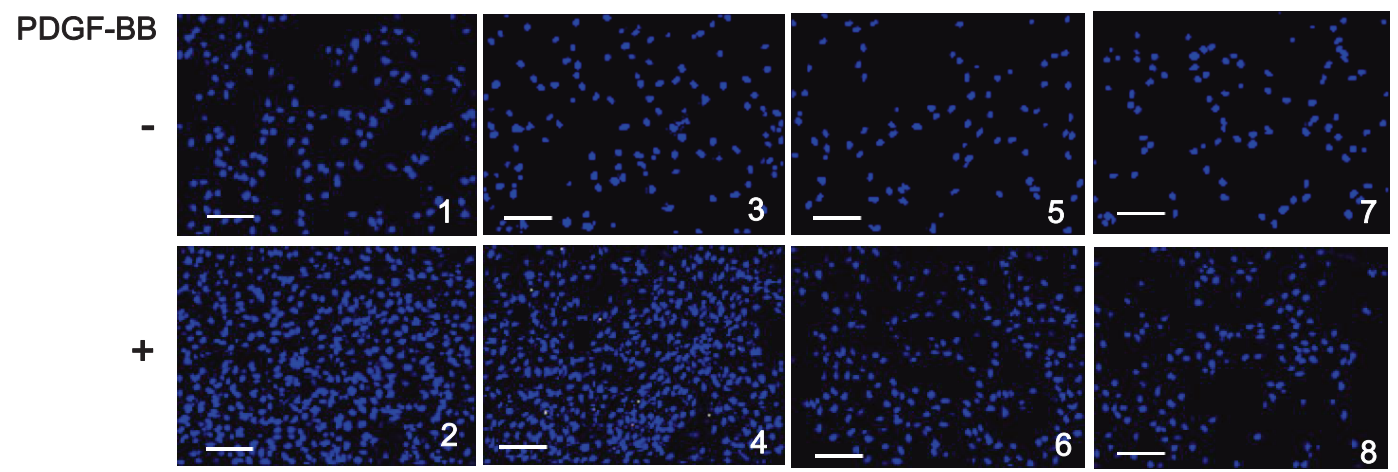

onalespib $(\mu \mathrm{M})$

0

0.1

0.3

0.5

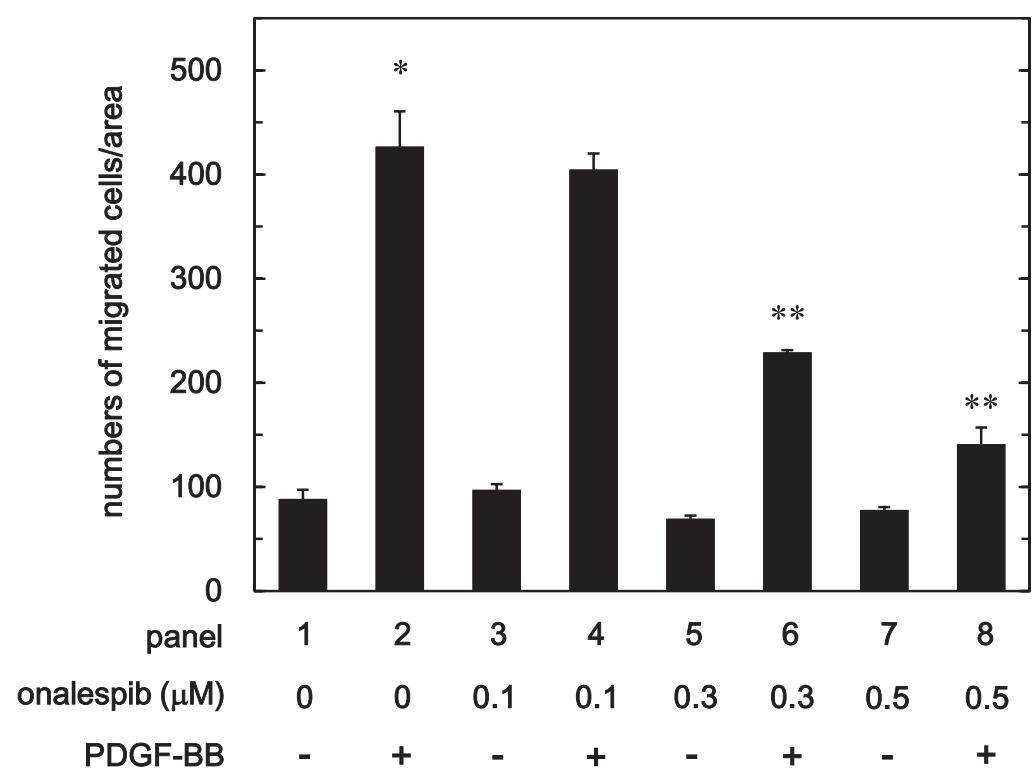

Fig. 1 Effect of onalespib on the PDGF-BB-induced migration of MC3T3-E1 cells. The cell migration was evaluated by a transwell cell migration assay. The cells were pretreated with various dose of onalespib for 60 min, and then stimulated by $3 \mathrm{ng} / \mathrm{mL}$ of PDGF-BB or vehicle for $16 \mathrm{~h}$. The cells were photographed by fluorescent microscopy at a magnification of $20 \times$, and the number of DAPI-stained cells in each field was counted. The upper panel shows the representative image of fluorescent microscopy. The lower panel shows a histogram indicating the number of migrated cells. Each value represents the mean \pm SEM of triplicate determinations from three independent cell preparations. ${ }^{*} P<0.05$, compared to the value of the control cells without PDGF-BB-stimulation. ${ }^{* *} P<0.05$, compared to the value of PDGF-BB alone. Scale bar; $100 \mu \mathrm{m}$.

significantly suppressed by geldanamycin $(0.3 \mu \mathrm{M})$, which caused about $75 \%$ reduction in the effect of PDGF-BB (Fig. 4).

Effects of onalespib on the PDGF-BB-stimulated phosphorylation of p38 MAP kinase, JNK or p44/ p42 MAP kinase in MC3T3-E1 cells

As for the intracellular signaling system of PDGF$\mathrm{BB}$ in osteoblast migration, we have demonstrated that p38 MAP kinase, JNK and p44/p42 MAP kinase play as positive regulators in the PDGF-BB-stimulated migration of MC3T3-E1 cells (8). In order to investigate how HSP90 inhibitors suppressed the
PDGF-BB-stimulated migration of these cells, we examined the effects of onalespib on the PDGF-BBinduced phosphorylation of p38 MAP kinase, JNK or p44/p42 MAP kinase. Onalespib failed to suppress the PDGF-BB-induced phosphorylation of $\mathrm{p} 38$ MAP kinase (Fig. 5A) or JNK (Fig. 5B). On the other hand, the PDGF-BB-induced phosphorylation of p44/p42 MAP kinase was significantly attenuated by onalespib (Fig. 6). 


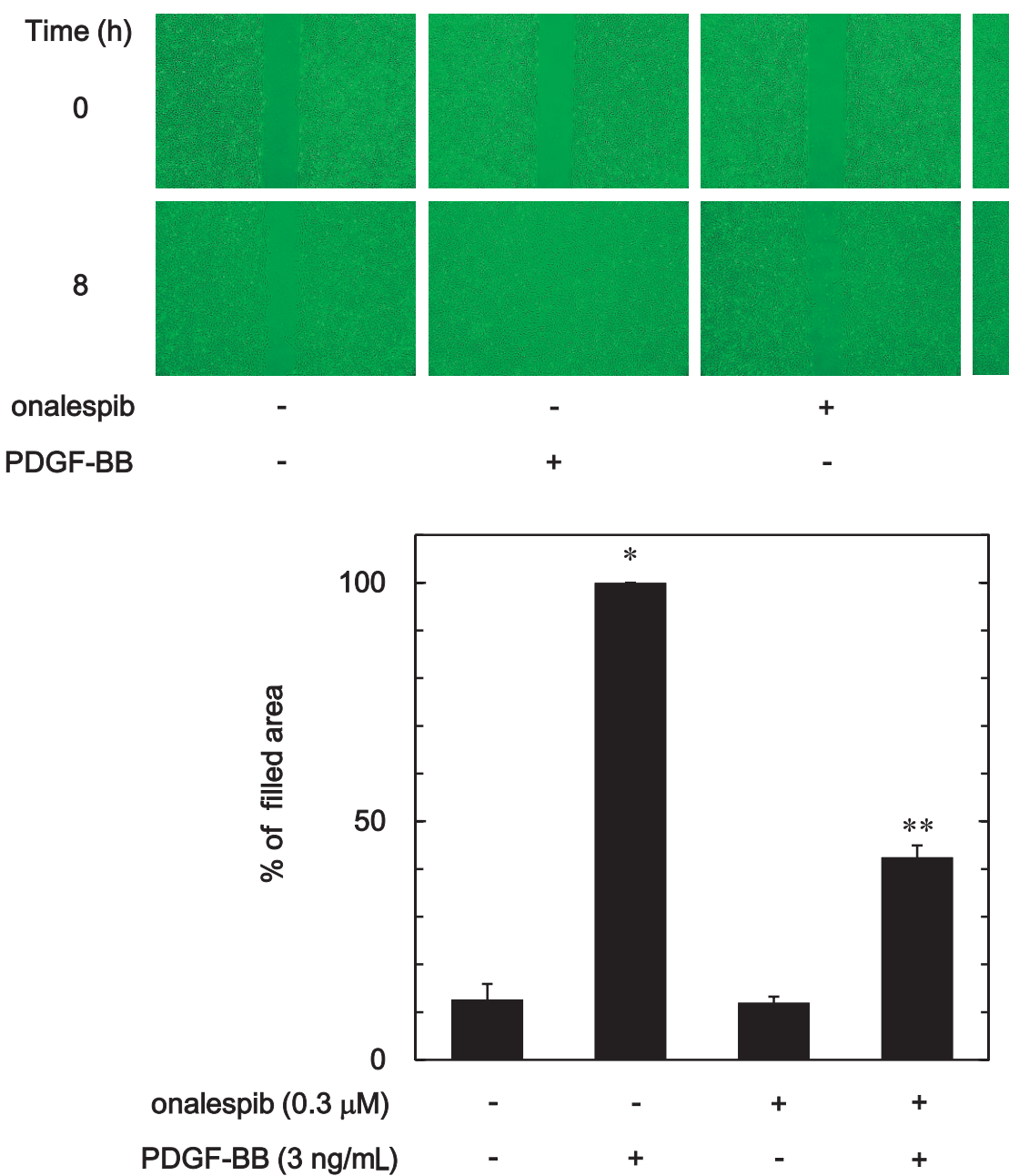

Fig. 2 Effect of onalespib on the PDGF-BB-induced migration of MC3T3-E1 cells. The cell migration was evaluated by a wound-healing assay. The cells were pretreated with $0.3 \mu \mathrm{M}$ of onalespib or vehicle for $60 \mathrm{~min}$, and then stimulated by $3 \mathrm{ng} /$ $\mathrm{mL}$ of PDGF-BB or vehicle for $8 \mathrm{~h}$. The cells were photographed before PDGF-BB-stimulation $(0 \mathrm{~h})$ and after $8 \mathrm{~h}$ (upper panel), and the area of migrated cells was measured (lower panel). Each value represents the mean \pm SEM of triplicate determinations from three independent cell preparations. ${ }^{*} P<0.05$, compared to the value of the control cells without PDGF-BB-stimulation. ${ }^{* *} P<0.05$, compared to the value of PDGF-BB alone.

Effect of geldanamycin on the PDGF-BB-stimulated phosphorylation of p44/p42 MAP kinase in MC3T3E1 cells

We additionally examined the effect of geldanamycin, another HSP90 inhibitor (18), on the PDGF-BBinduced phosphorylation of p44/p42 MAP kinase in MC3T3-E1 cells. Geldanamycin as well as onalespib remarkably decreased the levels of phosphorylated p44/p42 MAP kinase in a dose dependent manner between 0.3 and $1.0 \mu \mathrm{M}$ (Fig. 7).

\section{DISCUSSION}

It is firmly established that HSP90 is an ATP-dependent molecular chaperone (20). Onalespib and geldanamycin have been developed as HSP90 inhibitors, which interfere with the ATP binding site of HSP90, resulting in the suppression of chaperone activity $(3,18)$. In the present study, we investigated the implication in the PDGF-BB-induced migration of osteoblast-like MC3T3-E1 cells. We first examined the effect of onalespib (3) on the PDGF-BB-induced migration using a Boyden chamber, and found that onalespib significantly reduced the PDGF-BBinduced migration of MC3T3-E1 cells. Evaluated by a wound-healing assay, another assay for cell migration, we demonstrated that the cell migration induced by PDGF-BB was suppressed by onalespib. Therefore, these results suggest that HSP90 is involved in the regulation of PDGF-BB-stimulated 


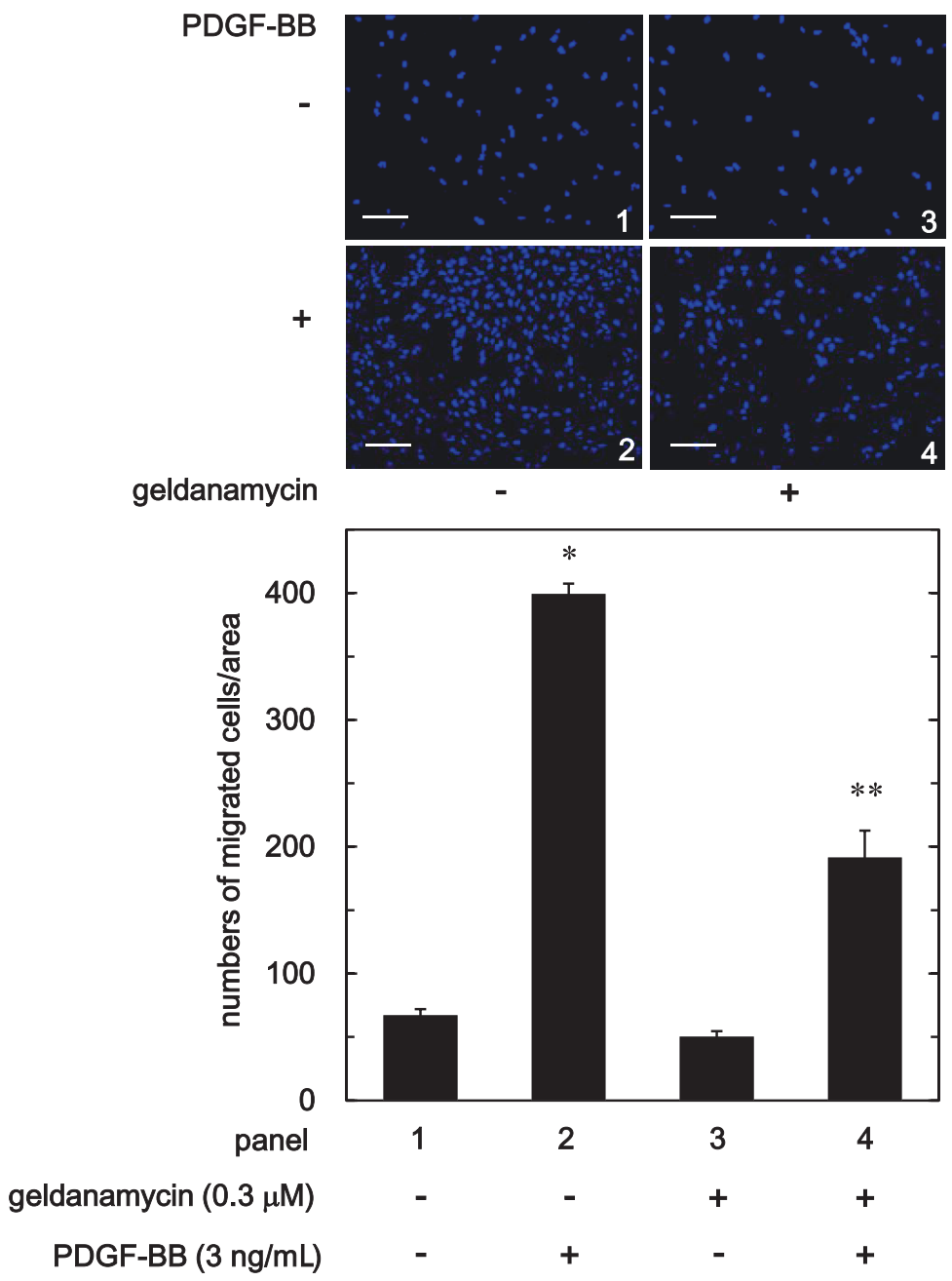

Fig. 3 Effect of geldanamycin on the PDGF-BBinduced migration of MC3T3-E1 cells. The cell migration was evaluated by a transwell cell migration assay. The cells were pretreated with $0.3 \mu \mathrm{M}$ of geldanamycin or vehicle for $60 \mathrm{~min}$, and then stimulated by $3 \mathrm{ng} / \mathrm{mL}$ of PDGF-BB or vehicle for $16 \mathrm{~h}$. The cells were photographed by fluorescent microscopy at a magnification of $20 x$, and the number of DAPI-stained cells in each field was counted. The upper panel shows the representative image of fluorescent microscopy. The lower panel shows a histogram indicating the number of migrated cells. Each value represents the mean \pm SEM of triplicate determinations from three independent cell preparations. ${ }^{*} P<0.05$, compared to the value of the control cells without PDGF-BB-stimulation. ${ }^{* *} P<0.05$, compared to the value of PDGF-BB alone. Scale bar; $100 \mu \mathrm{m}$. migration of MC3T3-E1 cells. We further examined the effect of geldanamycin, another type inhibitor of HSP90 different from onalespib (18), on the PDGFBB-induced migration of MC3T3-E1 cells. The PDGF-BB-induced migration of MC3T3-E1 cells, evaluated by both a transwell cell migration assay and a wound-healing assay, was markedly weakened by geldanamycin. Based on these our findings, it is most likely that HSP90 positively regulates the PDGF-BB-induced migration of osteoblast-like MC3T3-E1 cells.

Regarding the intracellular signaling of PDGF-BB in the migration of osteoblasts, a recent study from our laboratory has demonstrated that PDGF-BB induces the migration of osteoblast-like MC3T3-E1 cells through the activation of p38 MAP kinase, SAPK/JNK and p44/p42 MAP kinase (8). In order to elucidate the mechanism underlying the suppressive effect of HSP90 inhibitors in the PDGF-BB-induced migration of osteoblasts, we examined the effects of onalespib on the PDGF-BB-stimulated phosphorylation of p38 MAP kinase, JNK and p44/ p42 MAP kinase in MC3T3-E1 cells. However, onalespib failed to inhibit the PDGF-BB-stimulated phosphorylation of p38 MAP kinase or JNK. On the contrary, the levels of phosphorylated p44/p42 MAP kinase upregulated by PDGF-BB was significantly reduced by onalespib. Therefore, it seems that HSP90 plays a role as a positive regulator in the PDGF-BB-elicited activation of p44/p42 MAP kinase in osteoblast-like MC3T3-E1 cells. Additionally, we demonstrated that geldanamycin markedly suppressed the phosphorylation of p44/p42 MAP kinase by PDGF-BB. Taking our present findings using HSP90 inhibitors into account, it is most likely that HSP90 positively regulates the PDGF-BB-induced migration of MC3T3-E1 cells by $\mathrm{p} 44 / \mathrm{p} 42$ MAP kinase. The potential mechanism behind the suppressive effect of HSP90 inhibitors on the PDGF$\mathrm{BB}$-induced migration of osteoblasts is summarized 


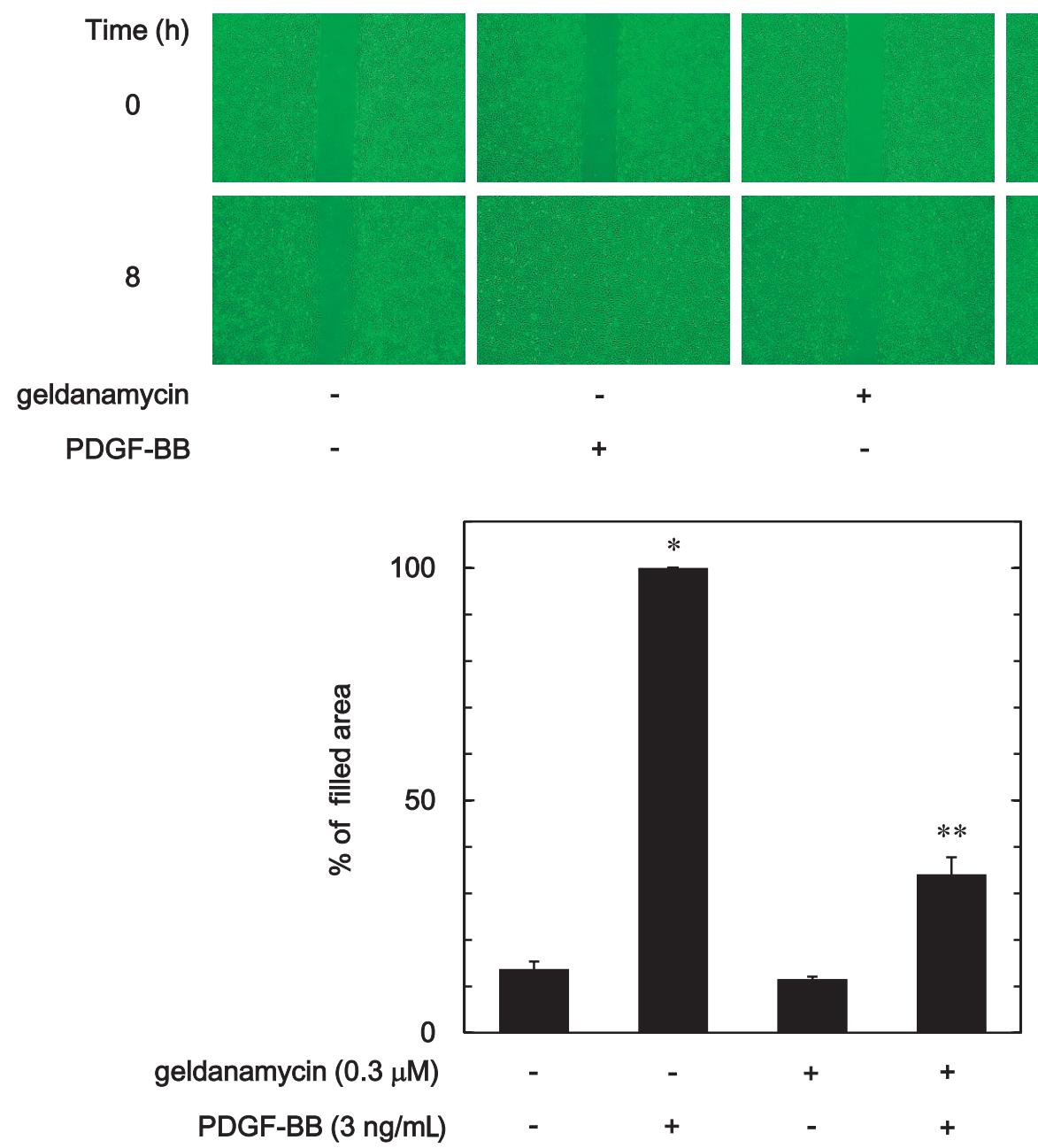

Fig. 4 Effect of geldanamycin on the PDGF-BB-induced migration of MC3T3-E1 cells. The cell migration was evaluated by a wound-healing assay. The cells were pretreated with $0.3 \mu \mathrm{M}$ of geldanamycin or vehicle for 60 min, and then stimulated by $3 \mathrm{ng} / \mathrm{mL}$ of PDGF-BB or vehicle for $8 \mathrm{~h}$. The cells were photographed before PDGF-BB-stimulation $(0 \mathrm{~h})$ and after $8 \mathrm{~h}$ (upper panel), and the area of migrated cells was measured (lower panel). Each value represents the mean \pm SEM of triplicate determinations from three independent cell preparations. ${ }^{*} P<0.05$, compared to the value of the control cells without PDGF-BB-stimulation. ${ }^{* *} P<0.05$, compared to the value of PDGF-BB alone.

as Fig. 8. The main pathway of PDGF-BB-induced migration of osteoblasts remains to be elucidated. Further investigations would be necessary to clarify the exact mechanism behind HSP90-effects on osteoblast functions.

In bone remodeling, osteoblasts migrate onto the bone lacuna resorbed by osteoclasts and the migrated osteoblasts subsequently initiate the formation of bone at the sites (11). It is generally recognized that adequate migration of osteoblasts is indispensable not only for the maintenance of physiological bone remodeling but also for the protection against pathological conditions including the distress of bone fracture repair and osteoporosis $(11,21)$. On the other hand, it is firmly established that under stress conditions, HSP90 is a major molecular chaperone which facilitates unfolded proteins to fold properly in an ATP-dependent manner (20). In addition, HSP90 has been considered to be a potent target for cancer therapy, and various HSP90 inhibitors have been developed as anti-cancer agents since HSP90 stabilizes proteins necessary for progression of cancer cells (20). It has been reported that knock-down of HSP90 suppresses the migration and proliferation of angiosarcoma cells (25). Accumulating evidence indicates that HSP90 is involved also in various physiological cellular functions such as regulation of glucocorticoid receptors (20). We have previously shown that HSP90 negatively regulates prostaglandin F2 $\alpha$-induced synthesis of interleukin- 6 , a potent 

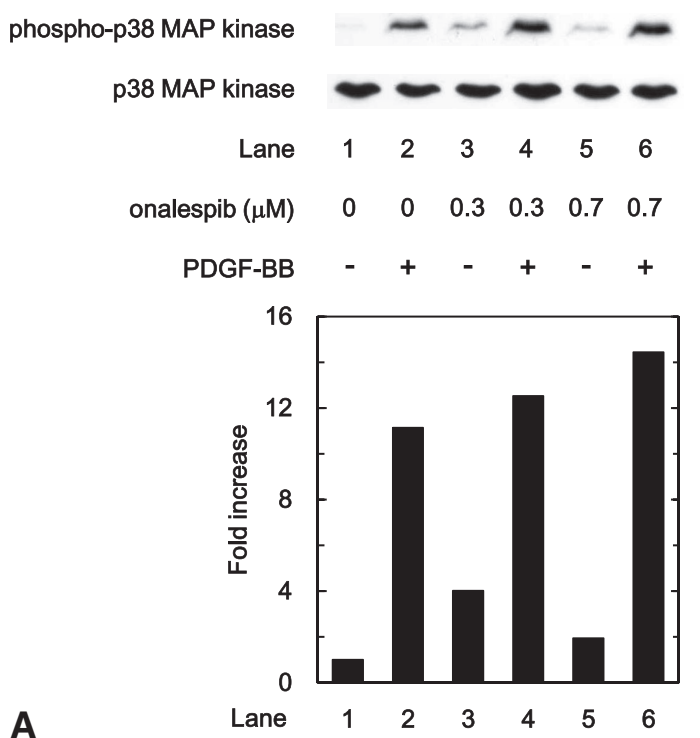
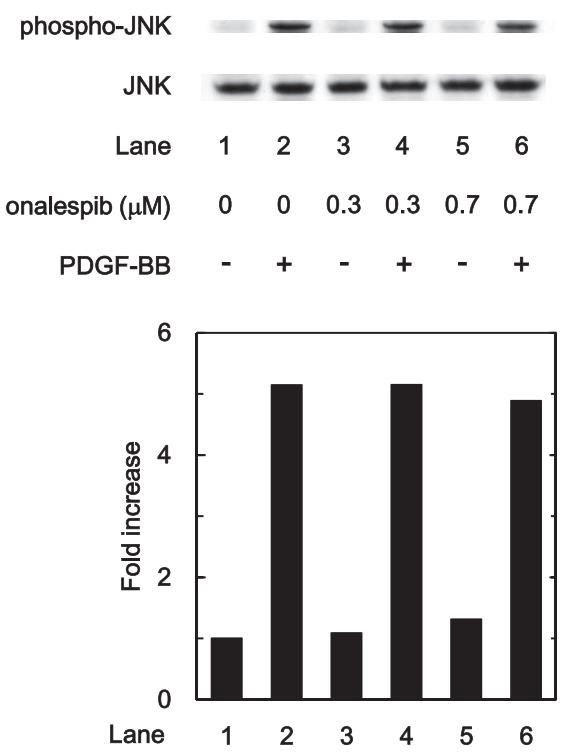

Fig. 5 Effect of onalespib on the PDGF-BB-induced phosphorylation of p38 MAP kinase (A) or JNK (B) in MC3T3-E1 cells. The cells were pretreated with the indicated doses of onalespib for $60 \mathrm{~min}$, and then stimulated by $30 \mathrm{ng} / \mathrm{mL}$ of PDGF-BB or vehicle for $10 \mathrm{~min}$. Western blot analysis was performed using antibodies against phospho-specific p38 MAP kinase, p38 MAP kinase, phospho-specific JNK or JNK. The histogram shows the quantitative representation of the PDGF-BB-induced levels from a laser densitometoric analysis. Each value is a representative of three independent cell preparations.
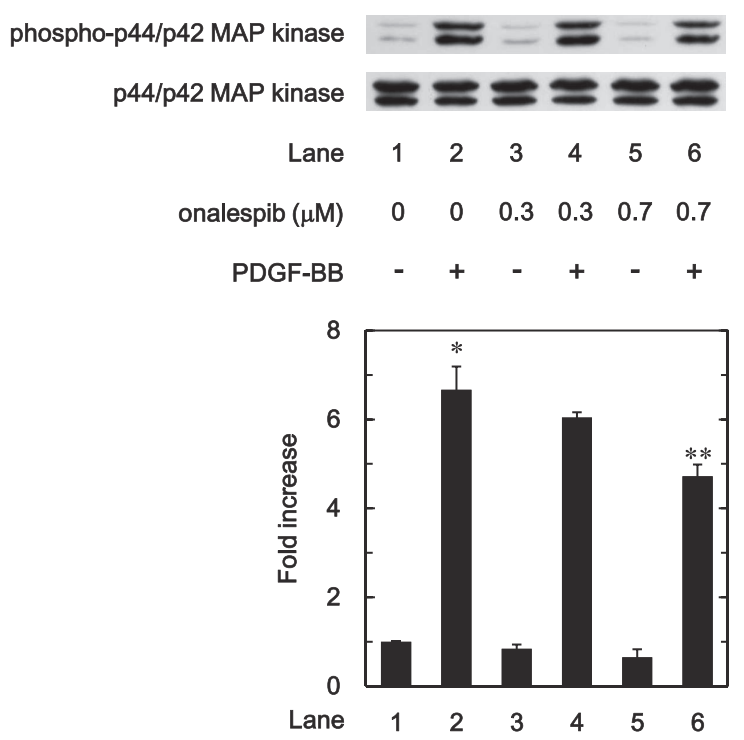

Fig. 6 Effects of onalespib on the PDGF-BB-induced phosphorylation of p44/p42 MAP kinase in MC3T3-E1 cells. The cells were pretreated with the indicated doses of onalespib for $60 \mathrm{~min}$, and then stimulated by $30 \mathrm{ng} / \mathrm{mL}$ of PDGF-BB or vehicle for $2 \mathrm{~min}$. Western blot analysis was performed using antibodies against phospho-specific p44/p42 MAP kinase or p44/p42 MAP kinase. The histogram shows the quantitative representations of the PDGF-BB-induced levels from a laser densitometoric analysis. Each value represents the mean \pm SEM of triplicate determinations from three independent cell preparations. ${ }^{*} P<0.05$, compared to the value of the control cells without PDGF-BB-stimulation. ${ }^{* *} P<0.05$, compared to the value of PDGF-BB alone.
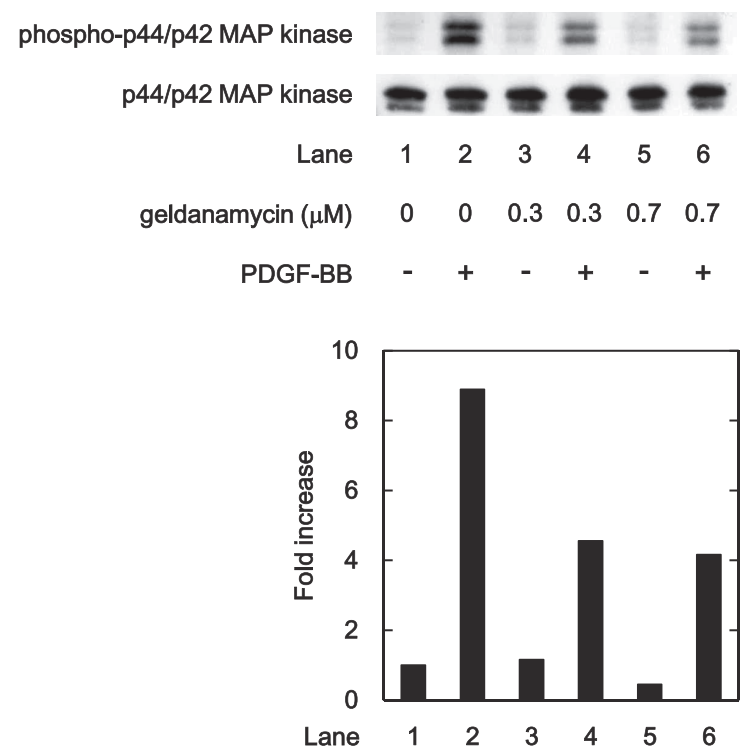

Fig. 7 Effect of geldanamycin on the PDGF-BB-induced phosphorylation of p44/p42 MAP kinase in MC3T3-E1 cells. The cells were pretreated with various doses of geldanamycin for $60 \mathrm{~min}$, and then stimulated by $30 \mathrm{ng} / \mathrm{mL}$ of PDGF$\mathrm{BB}$ or vehicle for $3 \mathrm{~min}$. Western blot analysis was performed using antibodies against phospho-specific p44/p42 MAP kinase or p44/p42 MAP kinase. The histogram shows the quantitative representation of the PDGF-BB-induced levels from a laser densitometoric analysis. Each value is a representative of three independent cell preparations. 


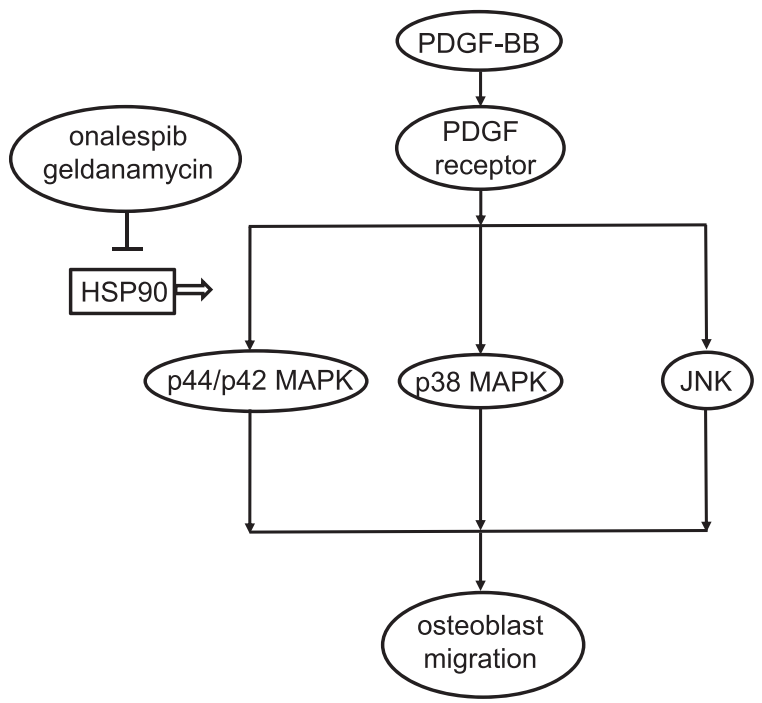

Fig. 8 Diagram of the potential mechanism behind the suppressive effect of HSP90 inhibitors on the PDGF-BB-induced migration of osteoblast-like MC3T3-E1 cells. PDGF, platelet-derived growth factor; MAPK, mitogen-activated protein kinase; JNK, c-Jun N-terminal kinase; HSP90, heat shock protein 90.

bone remodeling agent, in osteoblast-like MC3T3-E1 cells (4). Thus, HSP90 is currently recognized as a central regulator of proteostasis in a variety of cellular processes including cell proliferation and development (20). Additionally, HSP90 inhibitors are potentially identified as a novel class of senolytic drugs to anti-aging (5). In the present study, we demonstrated that HSP90 inhibitors reduced the PDGFBB-induced migration of osteoblast-like MC3T3-E1 cells, suggesting that HSP90 might play as a positive regulator in osteoblast migration. Based on our findings, it is probable that HSP90 is a potent therapeutic target for metabolic bone disease therapy such as osteoporosis, a major health problem in aging. Further investigations including the knock-down of HSP90 are required to investigate the implication of HSP90 in bone metabolism.

In conclusion, our results strongly suggest that HSP90 inhibitor diminishes the PDGF-BB-induced osteoblast migration through attenuation of $\mathrm{p} 44 / \mathrm{p} 42$ MAP kinase activity.

\section{Acknowledgements}

We are very grateful to Mrs. Yumiko Kurokawa for her skillful technical assistance. This investigation was supported in part by Grants-in-Aid for Scientific Research (15K10487 and 17K11002) from the Ministry of Education, Culture, Science, Sports and
Technology of Japan, and Research Funding for Longevity Sciences (28-9 and 29-12) from National Center for Geriatrics and Gerontology, Japan.

\section{DISCLOSURE}

The authors declare that they have no conflicts of interest.

\section{REFERENCES}

1. Canalis E (2009) Growth factor control of bone mass. J Cell Biochem 108, 769-777.

2. Caplan AI and Correa D (2011) PDGF in bone formation and regeneration: new insights into a novel mechanism involving MSCs. J Ortho Res 29, 1795-1803.

3. Ferraldeschi R, Welti J, Powers MV, Yuan W, Smyth T, Seed G, Riisnaes R, Hedayat S, Wang H, Crespo M, Nava Rodrigues D, Figueiredo I, Miranda S, Carreira S, Lyons JF, Sharp S, Plymate SR, Attard G, Wallis N, Workman P and de Bono JS (2016) Second-generation HSP90 inhibitor onalespib blocks mRNA splicing of androgen receptor variant 7 in prostate cancer cells. Cancer Res 76, 2731-2742.

4. Fujita K, Tokuda H, Kuroyanagi G, Yamamoto N, Kainuma S, Kawabata T, Sakai G, Matsushima-Nishiwaki R, Kozawa O and Otsuka T (2017) HSP90 inhibitors potentiate PGF2 $\alpha$ induced IL-6 synthesis via p38 MAP kinase in osteoblasts. PLoS One 12, e 0177878 .

5. Furmann-Stroissnigg H, Ling YY, Zhao J, McGwan SJ, Zhu Y, Brooks RW, Grassi D, Gregg SQ, Stripay LJ, Dorronsoro A, Corbo L, Tang P, Bukata C, Ring N, Giacca M, Li X, Tchkonia T, Kirkland JL, Niedernhofer LJ and Robbins PD (2017) Identification of HSP90 inhibitors as a novel class of senolytics. Nat Commun 8, 422.

6. Heldin CH and Westermark B (1999) Mechanism of action and in vivo role of platelet-derived growth factor. Physiol Rev 79, 1283-1316.

7. Hengartner N, Fiedler J, Ignatius A and Brenner RE (2013) IL-1 $\beta$ inhibits human osteoblast migration. Mol Med 19, 3642.

8. Kainuma S, Tokuda H, Yamamoto N, Kuroyanagi G, Fujita K, Kawabata T, Sakai G, Matsushima-Nishiwaki R, Kozawa O and Otsuka T (2017) Heat shock protein 27 (HSPB1) suppresses PDGF-BB-induced migration of osteoblasts. Int $J$ Mol Med 40, 1057-1066.

9. Kampinga HH, Hageman J, Vos MJ, Kubota H, Tanguay RM, Bruford EA, Cheetham ME, Chen B and Hightower LE (2009) Guidelines for the nomenclatures of the human heat shock proteins. Cell Stress Chaperones 14, 105-111.

10. Karagiosis SA, Chrisler WB, Bollinger $\mathrm{H}$ and Karin NJ (2009) Lysophosphatidic acid-induced ERK activation and chemotaxis in MC3T3-E1 preosteoblasts are independent of PDGF-BB receptor transactivation. J Cell Physiol 219, 716723.

11. Karsenty G and Wagner EF (2002) Reaching a genetic and molecular understanding of skeletal development. Dev Cell 2, 389-406.

12. Kato $\mathrm{K}$, Ito $\mathrm{H}$, Hasegawa $\mathrm{K}$, Inaguma $\mathrm{Y}$, Kozawa $\mathrm{O}$ and Asano T (1996) Modutation of the stress-induced synthesis of hsp27 and $\alpha$ B-crystallin by cyclic AMP in C6 rat glioma cells. J Neurochem 66, 946-950.

13. Kozawa O, Tokuda H, Miwa M, Kotoyori J and Oiso Y (1992) 
Cross-talk regulation between cyclic-AMP production and phosphoinositide hydrolysis induced by prostaglandin $\mathrm{E}_{2}$ in osteoblast-like cells. Exp Cell Res 198, 130-134.

14. Kozawa O, Niwa M, Hatakeyama D, Tokuda H, Oiso Y, Matsuno M, Kato K and Uematsu T (2002) Specific induction of heat shock protein 27 by glucocorticoid in osteoblasts. J Cell Biochem 86, 357-364.

15. Kular J, Tickner J and Chim SM (2012) An overview of the regulation of bone remodeling at the cellular level. Clin Biochem $45,863-873$.

16. Laemmli UK (1970) Cleavage of structural proteins during the assembly of the head of bacteriophage T4. Nature 227, 680-685.

17. Mori M, Hitora T, Nakamura O, Yamagami Y, Horie R, Nishimura H and Yamamoto T (2015) Hsp90 inhibitor induces autophagy and apoptosis in osteosarcoma cells. Int $J$ Oncol 46, 47-54.

18. Ochel HJ, Eichhorn K and Gademann G (2001) Geldanamycin: the prototype of a class of antitumor drugs targeting the heat shock protein 90 family of molecular chaperones. Cell Stress Chaperones 6, 105-112.

19. Price JT, Quinn JM, Sims NA, Vieusseux J, Waldeck K, Docherty SE, Myers D, Nakamura A, Waltham MC, Gillespie MT and Thompson EW (2005) The heat shock protein 90 in- hibitor, 17-allylamino-17-demethoxygeldanamycin, enhances osteoclast formation and potentiates bone metastasis of a human breast cancer cell line. Cancer Res 65, 4929-4938.

20. Schopf FH, Biebl MM and Buchner J (2017) The HSP90 chaperone machinery. Nat Rev Mol Cell Biol 18, 345-360.

21. Shah P, Keppler L and Rutkowski J (2014) A review of platelet derived growth factor playing pivotal role in bone regeneration. J Oral Implantol 40, 330-340.

22. Sims NA and Gooi JH (2008) Bone remodeling: Multiple cellular interactions required for coupling of bone formation and resorption. Semin Cell Dev Biol 19, 444-451.

23. Su P, Tian Y, Yang C, Ma X, Wang X, Pei J and Qian A (2018) Mesenchymal stem cell migration during bone formation and bone diseases therapy. Int J Mol Sci 19, E2343.

24. Sudo H, Kodama H, Amagai Y, Yamamoto S and Kasai S (1983) In vitro differentiation and calcification in a new clonal osteogenic cell line derived from newborn mouse calvaria. $J$ Cell Biol 96, 191-198.

25. Yamada-Kanazawa S, Fukushima S, Jinnin M, Masuzawa M, Maszawa M, Amoh Y, Hoshina D, Abe R and Ihn H (2017) Inhibition of heat shock protein 90 exerts an antitumor effect in angiosarcoma: involvement of the vascular endothelial growth factor signalling pathway. Br J Dermatol 177, 456469 . 